\title{
Ischemic and Non-ischemic Stroke in Young Adults - A Look at Risk Factors and Outcome in a Developing Country
}

Mohammed Tahar Si Larbi ${ }^{1}$, Waleed al Mangour ${ }^{1}$, Iram Saba ${ }^{2}$, Dhekra Al Naqeb ${ }^{3}$, Zaina Swapna Faisal ${ }^{1}$, Sana Omar $^{1}$, Fatima Ibrahim ${ }^{1}$

1. Medical Affairs, Sultan Bin Abdulaziz Humanitarian City, Riyadh, SAU 2. Research, Sultan Bin Abdulaziz Rehabilitation Center Riyadh, Riyadh, SAU 3. Research and Scientific Center, Sultan Bin Abdulaziz Humanitarian City, Riyadh, SAU

Corresponding author: Iram Saba, iramsabawani@gmail.com

\section{Abstract}

\section{Objective}

Stroke among young adults is the leading cause of disability worldwide. Efforts are being taken to control stroke in the general population, but in parallel, there is an increasing trend of stroke among the young population. These patients are often affected by physical disability, cognitive impairment, and loss of productivity, all of which have personal, social, and economic implications. The main aim of this study was to determine the risk factors associated with stroke among young patients admitted to a tertiary care rehabilitation center and determine the effect of rehabilitation on the outcome of their daily life activities.

\section{Materials and Methods}

A retrospective hospital-based cohort study was conducted between January 2015 to December 2019. Prevalence of stroke-related risk factors like hypertension, hyperlipidemia, diabetes, and cardiac disease was assessed.

\section{Results}

Out of 710 young stroke adults, $71.97 \%$ were described as ischemic, and $28.03 \%$ reported as non-ischemic. Mean age (SD) was found to be $44.54 \pm 9.3$. Univariate analysis demonstrated that hyperlipidemia, cardiac disease, and diabetes indicated a significantly higher risk for ischemic stroke with an OR (95\% CI) at 2.5 (1.73.7), 2.11 (1.2-3.6), and 1.66 (1.2-2.3) respectively. A significant improvement was observed in their Functional Independence Measure (FIM0 score after their rehabilitation irrespective of age and gender.

\section{Conclusion}

Review began 07/25/2021 Review ended 07/28/2021 Published 08/11/2021

\section{๑) Copyright 2021}

Si Larbi et al. This is an open access article distributed under the terms of the Creative Commons Attribution License CC-BY 4.0., which permits unrestricted use, distribution, and reproduction in any medium, provided the original author and source are credited.
Association of risk factors associated with stroke should be subjected to close follow-up and management, thus reducing the risk of developing long-lasting disabilities at a young age. The identification of risk factors for young stroke incidence is a step towards improving health in the young adult population.

Categories: Neurology, Public Health, Health Policy

Keywords: young adults, ischemic stroke, risk factors, rehabilitation, functional independence measure (fim)

\section{Introduction}

Stroke is a common neurological disorder increasing worldwide with 15 million strokes and 5.8 million stroke-related deaths per year [1]. The majority of incident cases of stroke are ischemic stroke (87\%), followed by intracerebral hemorrhage (10\%) and subarachnoid hemorrhage (3\%) [2]. Stroke in young adults is more heterogeneous than stroke in older persons, owing to the wide range of underlying risk factors and etiology. Despite an increasing number of young stroke patients, the risk factors of stroke remain unknown in about one-third of all patients [3]. The disturbing patterns of stroke in young adults are likely due to growing rates of modifiable risk factors such as hypertension, hyperlipidemia, obesity, and diabetes, highlighting the importance of early detection and active prevention measures in the general population at an early age [4]. Stroke among young patients has special attention since they have shown better recovery. This is different from stroke seen in developed countries, where a majority of stroke cases affect the older population.

The stroke specialty program of Sultan-Bin-Abdul-Aziz Humanitarian City (SBAHC), Riyadh, Saudi Arabia, provides individualized patient-centered, coordinated, and integrated services. The program had focused on different age-specific factors such as pregnancy, oral contraceptive use, and behavioral factor of the young population such as low physical activity and smoking. In developing countries, there is a lack of data on stroke among young patients despite such problems are rapidly growing; however, research in such areas is 
essential to plan for appropriate screening, diagnosis, and management. It is difficult and often subjective to identify an age cut-off, but young adults are usually identified as those under 55 years old in previously published studies and registries [5].

This retrospective hospital-based study aims to assess the different stroke types and risk factors of stroke in young patients and to assess post-stroke complications, outcomes, and activities of daily living using the Functional Independent Measure (FIM) instrument.

\section{Materials And Methods \\ Study population}

This is a retrospective hospital-based cohort study for patients diagnosed with stroke admitted to the stroke unit at Sultan bin Abdul Aziz Humanitarian city (SBAHC) for a rehabilitation program between January 2015 to December 2019. The inclusion criteria included the patients having a history of stroke for at least 6 months and aged between 18 to 55 years for both genders. The patients with a history of intracranial congenital diseases, malignancy, neurological diseases, and psychiatric illnesses were excluded. All the involved cases were classified as per the International Classification of Diseases, Tenth Revision (ICD-10) [6]. Ischemic stroke is an episode of neurological dysfunction caused by focal cerebral, spinal, or retinal infarction. Intracerebral hemorrhage is a focal collection of blood within the brain parenchyma or ventricular system that is not caused by trauma, while stroke, which is not otherwise specified, is defined as an episode of acute neurological dysfunction presumed to be caused by ischemia or hemorrhage, persisting for $\geqslant 24$ hours or until death.

\section{Data collection}

The data was collected using the case report form (CRF) that was pre-tested for the feasibility of the data collection process. The CRF was completed with the socio-demographic data (age, gender, and residence area), clinical assessment history of diseases (such as ischemic non-ischemic, hypertension, hyperlipidemia, cardiac disease, and family history of hypertension and diabetes) were also included. Smoking history was collected. Laboratory data include hemoglobin, white blood cells (WBC), and platelets in addition to metabolic markers, i.e., fasting and random blood sugar, HbA1c, lipid profile, including total cholesterol, low-density lipoprotein (LDL), and low-density lipoprotein (HDL) in addition to urea, creatinine, $\mathrm{Na}$, and K.

To assess the risk factors of stroke in young patients and post-stroke complications and outcomes in activities of daily life, a Functional independent measurement (FIM) [7] instrument was used. The FIM Score is a 126-point instrument that comprises 18 individual subscales measuring a variety of physical and cognitive functions. Each subscale is scored from 1 to 7 ( 1 = total assist, $7=$ complete independence), resulting in a total FIM score that ranges from 18 to 126 . The FIM is well known and validated in the rehabilitation and stroke populations. Permission to use the FIM in this study was granted under the Medical Rehabilitation program of SBAHC. The study was approved by the Institutional Review Board of Sultan bin Abdul Aziz Humanitarian city (SBAHC), Riyadh, Kingdom of Saudi Arabia.

\section{Statistical Analysis}

The chi-square test and Mann-Whitney $\mathrm{U}$ test were used to analyze the categorical and continuous data respectively. The relationship between risk factors and stroke type was examined by univariate and agegender adjusted logistic regression. Odds ratios (with 95\% confidence interval) were used for assessing stroke risk factors using the result of univariate analysis, while age and gender-adjusted models for logistic regression analysis were used to control any confounders. Statistically significant was consistent if $\mathrm{p}$-value $<0.05$.

\section{Results}

\section{Characteristics of the study population}

The study included 710 young stroke patients (18- 55 years) who fulfill the criteria of stroke and have been assessed for risk factors. Out of the total number, 511 (71.97\%) were described as an ischemic type, and 199 (28.03\%) were reported as non-ischemic type (hemorrhage or others). Table 1 demonstrates the demographic characteristics for 480 (67.6\%) male and 230 (32.4\%) females with mean age (SD) of 44.74 (9.39) and 44.03 (8.95) respectively.

\begin{tabular}{|c|c|c|c|c|}
\hline Variables & All & Ischemic stroke & Non-ischemic stroke & P-value \\
\hline Number & 710 & $511(71.97)$ & $199(28.03)$ & \\
\hline Age (years) & $44.54 \pm 9.27$ & $44.74 \pm 9.39$ & $44.03 \pm 8.95$ & 0.35 \\
\hline Male & $480(67.6)$ & 347 (72.3) & $133(27.7)$ & 0.79 \\
\hline
\end{tabular}




\section{Cureus}

\begin{tabular}{|c|c|c|c|c|}
\hline Female & 230 (32.4) & $164(71.3)$ & $66(28.7)$ & 0.78 \\
\hline Currently employed & $111(41.3)$ & 77 (69.4) & $34(30.6)$ & 0.56 \\
\hline Currently unemployed & $158(58.7)$ & $109(69.0)$ & 49 (31.0) & 0.366 \\
\hline Living in urban area & 550 (77.5) & 395 (71.8) & 155 (28.2) & 0.92 \\
\hline Living in rural area & $160(22.5)$ & $116(72.5)$ & $44(27.5)$ & 0.92 \\
\hline Smoking & $94(4.1)$ & $67(71.3)$ & $27(28.7)$ & 0.90 \\
\hline Weight (kg) & $76.16 \pm 17.23$ & $75.90 \pm 16.87$ & $76.81 \pm 18.16$ & 0.51 \\
\hline Height (cm) & $164.63 \pm 13.54$ & $164.52 \pm 14.68$ & $164.92 \pm 10.04$ & 0.64 \\
\hline $\mathrm{BMI}\left(\mathrm{kg} / \mathrm{m}^{2}\right)$ & $23.16 \pm 5.07$ & $23.11 \pm 5.06$ & $23.28 \pm 5.11$ & 0.69 \\
\hline Systolic blood pressure & $120.46 \pm 17.48$ & $120.49 \pm 17.99$ & $120.38 \pm 16.1$ & 0.94 \\
\hline Diastolic blood pressure & $75.7 \pm 9.66$ & $75.44 \pm 9.56$ & $76.38 \pm 9.91$ & 0.24 \\
\hline Hypertension & 442 (62.3) & $308(69.7)$ & $134(30.3)$ & 0.085 \\
\hline Diabetes & 308 (43.3) & 239 (77.6) & $69(22.4)$ & $<0.001$ \\
\hline Hyperlipidemia & 247 (34.7) & $205(83.0)$ & $42(17.0)$ & 0.003 \\
\hline Cardiac disease & $112(15.8)$ & $93(83.0)$ & $19(17.0)$ & 0.004 \\
\hline Blood hemoglobin & $13.3 \pm 6.3$ & $13.3 \pm 7.06$ & $13.11 \pm 3.76$ & 0.60 \\
\hline WBC & $6.83(5.5-8.6)$ & $6.85(5.5-8.8)$ & $6.8(5.5-8.3)$ & 0.034 \\
\hline Platelets & $264(224-321)$ & 264 (228 - 224) & $260(219-314)$ & 0.096 \\
\hline Fasting blood sugar & $5.37(3.1-6.3)$ & $5.4(4.9-6.65)$ & $5.3(4.86-5.94)$ & 0.49 \\
\hline Random blood sugar & $6.1(5-9.4)$ & $6.5(5.17-9.8)$ & $5.6(4.9-7.6)$ & 0.95 \\
\hline HbA1c & $5.9(5.3-7.2)$ & $6.1(5.4-7.4)$ & $5.5(5.2-6.3)$ & 0.60 \\
\hline Urea & $5.3 \pm 2.8$ & $5.16 \pm 2.7$ & $5.53 \pm 3.06$ & 0.22 \\
\hline Serum creatinine & $66.6(54-80)$ & $66.4(54.3$ - 80.4) & $67(55.9$ - 82.8) & 0.50 \\
\hline Serum sodium & $141.69 \pm 58.8$ & $142.53 \pm 64.8$ & $139.4 \pm 38.79$ & 0.49 \\
\hline Serum potassium & $4.03 \pm 0.84$ & $4.05 \pm 0.75$ & $3.97 \pm 1.03$ & 0.07 \\
\hline Total cholesterol & $4.02 \pm 1.1$ & $3.94 \pm 1.1$ & $4.22 \pm 1.0$ & 0.002 \\
\hline LDL & $2.27 \pm 1$ & $2.19 \pm 1$ & $2.47 \pm 0.9$ & 0.32 \\
\hline HDL & $1.08 \pm 0.3$ & $1.07 \pm 0.3$ & $1.1 \pm 0.3$ & 0.82 \\
\hline Triglycerides & $1.37(1-1.8)$ & $1.38(1-1.8)$ & $1.36(1-1.8)$ & 0.86 \\
\hline
\end{tabular}

\section{TABLE 1: Study cohort clinical, hematological, metabolic, and biochemical characteristics}

Note: Data Presented as Mean \pm SD and Median (1st Q-3rd Q) for Gaussian and Non-Gaussian variables. P-value was calculated for differences between the two groups, and $p<0.05$ was considered as significant.

WBC - white blood cells, LDL - low-density lipoprotein, HDL - high-density lipoprotein

The study cohort did not demonstrate any clinical, biochemical, and hematological difference between ischemic and non-ischemic patients. Further analysis indicated that the proportion of patients suffering from diabetes, hyperlipidemia, and cardiac diseases were significantly higher in Ischemic vs. non-ischemic stroke (p-values of $<0.001,0.003$, and 0.004 , respectively). Total cholesterol levels were significantly lower among ischemic stroke $(3.94 \pm 1.11 \mathrm{mmol} / \mathrm{l}$ vs. $4.22 \pm 1.0 \mathrm{mmol} / \mathrm{l}, \mathrm{p}=0.002)$. 


\section{Cureus}

The prevalence of stroke increases with age regardless of type or gender except for the age group > 50 years for non-ischemic stroke in both genders and ischemic female group. There are more male stroke patients than females in any given age group. Figure 1 shows the highest stroke prevalence was among ischemic males aged $>50$ years, while the lowest was observed among non-ischemic females aged $<50$ years.

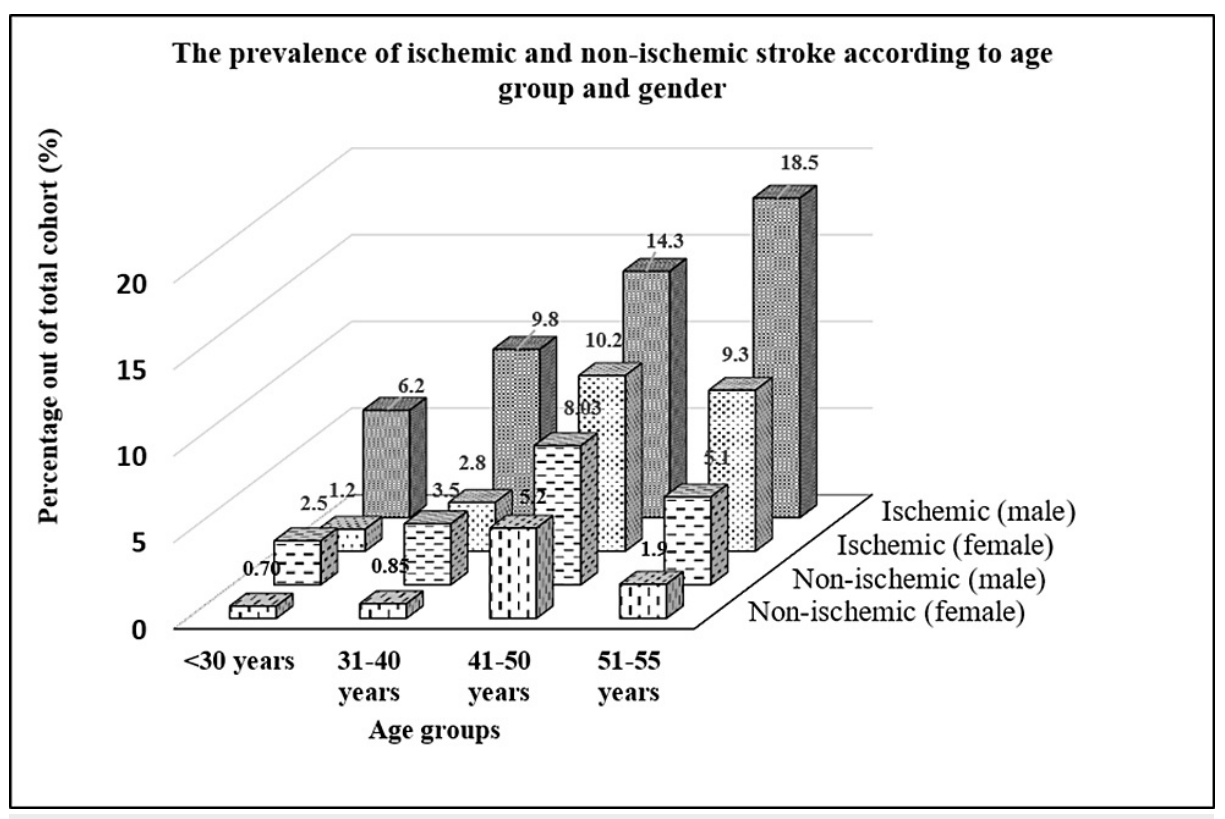

FIGURE 1: Distribution for Age and Gender in relation to stroke type

P-value was significant $(<0.001)$ for each age group between Ischemic and non-ischemic patients except for group $<30$ years in females $(p=0.91)$.

\section{Complications associated with stroke in the study population}

Analysis of the relationship between stroke-associated complications with that of gender, age, and etiology was presented in Table 2 .

\begin{tabular}{|c|c|c|c|c|c|c|c|c|c|c|}
\hline \multirow{2}{*}{ Variable } & \multirow{2}{*}{ Total (\%) } & \multicolumn{3}{|l|}{ Gender } & \multicolumn{3}{|l|}{ Age } & \multicolumn{3}{|l|}{ Etiology } \\
\hline & & Male & Female & Pivalue & $<30$ & $\geq 30$ & Pivalue & Ischemic & Non-ischemic & $\mathrm{P}$-value \\
\hline Seizure & 90 (12.70) & $58(64.40)$ & $32(35.60)$ & $<0.001$ & $21(23.30)$ & 69(76.70) & $<0.001$ & $60(66.60)$ & $30(33.40)$ & $<0.001$ \\
\hline Speech impairment & 90 (12.70) & $55(61.10)$ & $35(38.90)$ & 0.0028 & $23(25.50)$ & $67(74.50)$ & $<0.001$ & $64(71.20)$ & $26(28.80)$ & $<0.001$ \\
\hline Depression & $52(7.30)$ & $36(69.24)$ & $16(30.76)$ & $<0.001$ & $20(38.40)$ & $32(61.60)$ & 0.019 & $34(65.30)$ & $18(34.70)$ & $<0.001$ \\
\hline Urinary Incontinence & $46(6.50)$ & $32(69.56)$ & 14(30.44) & $<0.001$ & $12(26.80)$ & $34(73.20)$ & $<0.001$ & 29(63.10) & $17(36.90)$ & 0.01 \\
\hline Recurrent Stroke & $40(5.60)$ & $25(62.50)$ & 15(37.50) & 0.02 & $7(17.50)$ & $33(82.5)$ & $<0.001$ & $29(72.50)$ & $11(27.50)$ & $<0.001$ \\
\hline Constipation & $28(3.90)$ & $22(78.57)$ & $6(21.43)$ & $<0.001$ & 15(53.70) & 13(46.30) & $<0.001$ & 19(67.80) & $9(32.20)$ & $<0.001$ \\
\hline *Physical disabilities & $154(21.7)$ & $101(65.60)$ & $53(34.40)$ & $<0.001$ & $35(22.72)$ & 119(77.28) & $<0.001$ & $110(71.42)$ & $44(28.58)$ & $<0.001$ \\
\hline **Other complications & $60(8.30)$ & $42(70.00)$ & $18(30.00)$ & $<0.001$ & $8(13.34)$ & $52(86.66)$ & $<0.001$ & $40(66.67)$ & $20(33.33)$ & 0.0003 \\
\hline
\end{tabular}

TABLE 2: Distribution of complications associated with stroke in relation to age, gender, and types of strokes

Note: *Physical disabilities: mobility, speech, swallowing disabilities. ${ }^{\star *}$ Other complications: pulmonary embolism, thyroid disorder, bipolar syndrome, kidney disorder, anemia, cardiomyopathy.

Physical disabilities were found in $21.7 \%$ affecting more males older than 30 years mainly ischemic in 


\section{Cureus}

nature. The second most frequent complications were seizures and speech impairment at the rate of $12.70 \%$, which is significantly higher in males at an age older than 30 years and ischemic in etiology. The other complications were depression and urinary incontinence at the rate of $7.3 \%$ and $6.5 \%$, respectively.

The stroke recurrence was found to be at the rate of 5.6\% in this cohort and was more among males who were older than 30 years of age and had an ischemic stroke. The other associated complications, including mobility, speech, swallowing, and other impairments, were also significantly higher in ischemic male patients who were above $\geqslant 30$ of age.

\section{FIM scores before and after rehabilitation}

Table 3 demonstrates a significant increase in Functional Independent Score (FIM) score at discharge when compared with admission in genders, different age group, etiology, and pre-morbidity.

\begin{tabular}{|c|c|c|c|c|}
\hline \multirow{2}{*}{ Risk factors } & & \multicolumn{3}{|c|}{ FIM Score Mean \pm SD } \\
\hline & & Admission score & Discharge score & P-value \\
\hline \multirow{2}{*}{ Gender } & Male & $77.63 \pm 27.74$ & $94.28 \pm 25.96$ & $<0.001$ \\
\hline & Female & $77.7 \pm 27.71$ & $94.36 \pm 25.99$ & $<0.001$ \\
\hline \multirow{2}{*}{ Age } & $<30$ years & $87.09 \pm 22.48$ & $101.28 \pm 20.15$ & 0.0001 \\
\hline & $>30$ years & $76.5 / \pm 28.04$ & $93.50 \pm 26.42$ & $<0.001$ \\
\hline \multirow{2}{*}{ Etiology } & Ischemic & $71.12 \pm 27.69$ & $94.37 \pm 25.97$ & $<0.001$ \\
\hline & Non-Ischemic & $77.45 \pm 27.45$ & $94.17 \pm 26.02$ & $<0.001$ \\
\hline \multirow{4}{*}{ Pre-Morbidity } & Hypertensıon & $74.31 \pm 27.80$ & $94.30 \pm 26.00$ & $<0.001$ \\
\hline & Diabetes mellitus & $/ 2.44 \pm 28.00$ & $89.38 \pm 27.30$ & $<0.001$ \\
\hline & Hyperlipidemia & $76.92 \pm 26.70$ & $93.33 \pm 24.92$ & $<0.001$ \\
\hline & Cardiac Disease & $73.77 \pm 28.07$ & $91.01 \pm 26.46$ & $<0.001$ \\
\hline
\end{tabular}

\section{TABLE 3: FIM score before and after rehabilitation program for the total cohort.}

Note: P-value between different gender, etiology, and pre- morbidity were non-significant, while p-value was significant for age-group between FIM admission and Discharge Score.

FIM - Functional Independent Measure

Although there was no significant difference within those subgroups either during admission or discharge, a significant difference was found between FIM admission and FIM discharge in these groups.

\section{The adjusted odds ratio for important risk factors among the studied cohort}

The odds ratio for important risk factors has been given in Table 4 . 


\begin{tabular}{|c|c|c|c|c|c|c|c|c|}
\hline \multirow[b]{2}{*}{ Risk } & \multicolumn{5}{|l|}{ Univariate } & \multicolumn{3}{|c|}{ Age and gender adjusted } \\
\hline & Ischemic (511) & Non-Ischemic (199) & OR & $\mathrm{Cl}$ & P-value & OR & $\mathrm{Cl}$ & P-value \\
\hline Hyperlipidemia & $205(40.1)$ & $42(21.1)$ & 2.50 & $1.7-3.7$ & $<0.001$ & 2.54 & $1.7-3.7$ & $<0.001$ \\
\hline Cardiac disease & $93(18.2)$ & $19(9.5)$ & 2.11 & $1.2-3.6$ & 0.005 & 2.15 & $1.3-3.6$ & 0.004 \\
\hline Diabetes & $239(46.8)$ & $69(34.7)$ & 1.66 & $1.2-2.3$ & 0.004 & 1.64 & $1.2-2.3$ & 0.005 \\
\hline Hypertension & $308(60.3)$ & $134(67.3)$ & 0.14 & $0.5-1.1$ & 0.08 & 0.13 & $0.5-1.1$ & 0.07 \\
\hline Male gender & $347(67.9)$ & $133(66.8)$ & 1.05 & $0.7-1.5$ & 0.18 & - & - & - \\
\hline Age $\geq 45$ & $308(60.27)$ & $113(56.78)$ & 1.15 & $0.82-1.6$ & 0.39 & - & - & - \\
\hline
\end{tabular}

The univariate analysis in Table 4 demonstrated that hyperlipidemia, cardiac disease, and diabetes indicated a significantly higher risk for Ischemic stroke with OR (95\% CI) at 2.5 (1.7-3.7), 2.11 (1.2 - 3.6), and 1.66 (1.2 2.3) respectively. Hypertension, male gender, and age $\geqslant 45$ years remained as second most important risk factor, with OR ( $95 \% \mathrm{CI})$ at $0.74(0.5$ - 1.11), $1.05(0.7$ - 1.5) and $1.15(0.82$ - 1.6) respectively. hyperlipidemia, cardiac disease, and diabetes were also significant risk factors in age and gender-adjusted regression analyses.

\section{Discussion}

This large cohort study over a long period collected young stroked patients from wide areas in the Kingdom, being a tertiary rehabilitation center. In developing countries mean age of the first stroke is 63 years, compared to 69 in the US and 70 in England, which indicates that developing countries are hosting more young populations with stroke [8]. Although we are presenting non-acute stroke patients, this study had brought up a better understanding for patients suffering from ischemic stroke versus non-ischemic stroke in the young age group (18-55 years). Prevalence of stroke regardless of etiology demonstrates increment with age although males are three times more than females for ischemic stroke in different age groups, which is an observation reported from Helsinki Young Stroke Registry for age-specific stroke [9]. This could be related to the low prevalence of vascular changes observed in females based on their hormonal factors, in addition to the fact that males are prone to large vessel atherosclerosis at this age [10].

Non-ischemic stroke was more predominant among males, especially below 40 years, mainly in the form of a subarachnoid and cerebral hemorrhage. This is the same observation as found in an inter-stroke study that has investigated different risk factors for stroke involving 32 countries, including data from the middle east [11]. As males are more prone to trauma than females, we can see a higher incidence of hypertension among them which later contribute to intracerebral and subarachnoid hemorrhage [12].Other explanations like a higher incidence of arteriovenous malformation prevalence in males when compared with females [13].

This study age spectrum was between 18 to 55 years where there is an increase in the prevalence by $50 \%$ with any 10 years increment of the age. The drop-in prevalence of non-ischemic stroke is most likely related to a decrease rate of risk factors associated with non-ischemic strokes like trauma, congenital anomalies, and malignant hypertension [14]. Each year almost 11 million ischemic strokes occur globally, half of which occur in low and middle-income countries [15]. A significant cause of long-term impairment in this condition has a profound influence on patients and caregivers on the quality of life.

Although hypertension is the most frequent risk factor in both ischemic and non-ischemic patients, the univariate and age/gender-adjusted odds ratio did not demonstrate this risk factor to be of a significant value that is similar to the findings from a retrospective hospital-based study from Iran [16]. This could result from antihypertensive drug therapy use, as well as lifestyle modification changes among young adults. A metaanalysis study conducted by Law et al. showed that when the systolic and diastolic blood pressure was reduced by antihypertensive therapy, the incidence of stroke declined by $41 \%$ [17].

In the current study, we also found that hyperlipidemia is strongly associated with an ischemic stroke which is observed by Ansell et al., where they report the risk of ischemic stroke to be almost double that of nonischemic stroke [18]. The Strong Heart Study revealed that greater lipoprotein cholesterol level confers a high risk of ischemic stroke. High triglycerides and low HDL levels observed in patients suffering from diabetes have a more than two times higher incidence of ischemic stroke [19]. Although this study showed a lower value for total cholesterol and LDL as a result of therapeutic intervention, this will not eliminate the 
role of this important risk factor. In patients with large artery disease or disease of small vessels, hyperlipidemia is more frequently observed, while it is less common in ischemic patients having cardiac embolism [20]. In stroke due to large artery disease or small vessel disease, hyperlipidemia increases the risk among older adults who are often to have large artery disease and small-vessel disease due to stroke.

The studies have shown that diabetes among young adults is associated with a higher risk of stroke, and it's disturbing that the incidence of diabetes in young adults is increasing [21]. This study finds a significant association between diabetes and incidence of stroke; also, the univariate age and gender-adjusted odds ratio showed significant importance, particularly the ischemic stroke. This is inconsistent with other studies where diabetes is the main risk factor following hypertension and has been identified as a significant independent variable of symptomatic recurrence in patients with first-ever stroke [22]. This would stress the fact that glycemic control of patients with diabetes carries an important role in preventing ischemic stroke [23].

The other risk factor like cardiovascular disease also showed a significant univariate and age/genderadjusted odds ratio. This could result in atherosclerotic changes, especially in the left ventricle observed in both ischemic cardiac and cerebral events showing the frequency of ischemic stroke to be inversely proportional to left ventricular ejection fraction [24].

A total of $5.6 \%$ of the studied cohort were found to have a recurrent stroke. The other studies also reported the frequency of recurrent stroke between $2 \%$ to $18 \%$ [25]. The other stroke complications like seizures, depression, urinary incontinence, and constipation were found significantly higher in older male ischemic patients. A history of smoking was not significant, which could be due to a small number of smokers, especially among women in this community.

Obesity was not observed among stroked patients regardless of the type of stroke. This is inconsistent with the Northern Manhattan Study, which has concluded that abdominal obesity is an independent risk factor for ischemic stroke in all ethnic groups where BMI has no value on such findings [26].

FIM score is a very important tool for young stroke patients, not for their initial assessment but their degree of improvement. When looking at gender, age, etiology, and pre morbidity factors, there was a clear significant improvement which is expected in younger patients when compared with the older age group. Early intervention and treatment of patients with stroke not only decreases death but also decreases impairment and increases the enhancement of survival and independence [27].

Our study did not use the TOAST (Trial of Org 10172 in Acute Stroke Treatment) rating system to classify subtypes of acute ischemic stroke due to incompleteness to gather data. However, the above limitation does not compromise the essential data for case definition and major risk factors. The other limitation was that it excluded the ones having a history of intracranial congenital diseases, malignancy, neurological and psychiatric illness.

\section{Conclusions}

The study concludes that risk factors such as hypertension, hyperlipidemia, cardiac disease, and diabetes were more associated with an ischemic stroke rather than non-ischemic stroke, and this calls for a prevention plan at the national level with a focus on various lifestyle factors, proper rehabilitation process for young adults to prevent stroke and recommendations to help diagnose stroke at a young age and improve care. We are also looking forward to the implementation of a population-based study of stroke in each region and making ties with regional and national stroke registries here in Saudi Arabia. The identification of risk factors for young stroke incidence is a step towards improving health in young adults. Various organizations have disseminated awareness and encouraged research in this area. However, a more comprehensive study with larger numbers and well-defined risk factors is needed before such programs can be established. These types of treatments could lead to better treatment and management, potentially reducing the impact and burden of ischemic stroke in the highly productive age group.

\section{Additional Information \\ Disclosures}

Human subjects: Consent was obtained or waived by all participants in this study. Ethics Committee-Sultan Bin Abdulaziz Humanitarian City issued approval 4/67/175981. The study has been approved by the Ethics Committee at Sultan Bin Abdulaziz Humanitarian City, Riyadh, Saudi Arabia. Animal subjects: All authors have confirmed that this study did not involve animal subjects or tissue. Conflicts of interest: In compliance with the ICMJE uniform disclosure form, all authors declare the following: Payment/services info: All authors have declared that no financial support was received from any organization for the submitted work. Financial relationships: All authors have declared that they have no financial relationships at present or within the previous three years with any organizations that might have an interest in the submitted work. Other relationships: All authors have declared that there are no other relationships or activities that could appear to have influenced the submitted work. 


\section{Acknowledgements}

The authors would like to acknowledge the staff of the research center, nurses from the surgical department, and the department of health information system (HIS) in the city.

\section{References}

1. El-Haji M, Salameh P, Rachidi S, Hosseini H: The epidemiology of stroke in the Middle East . Eur Stroke J. 2016, 1:180-98. 10.1177/2396987316654338

2. Al-Senani F, Al-Johani M, Salawati M, et al.: An epidemiological model for first stroke in Saudi Arabia . J Stroke Cerebrovasc Dis. 2020, 29:104465. 10.1016/j.jstrokecerebrovasdis.2019.104465

3. van Alebeek ME, Arntz RM, Ekker MS, et al.: Risk factors and mechanisms of stroke in young adults: The FUTURE study. J Cereb Blood Flow Metab. 2018, 38:1631-41. 10.1177/0271678X17707138

4. Boehme AK, Esenwa C, Elkind MS: Stroke risk factors, genetics, and prevention . Circ Res. 2017, 120:472-95. 10.1161/CIRCRESAHA.116.308398

5. Lang MJ, David V, Giese-Davis J: The age conundrum: a scoping review of younger age or adolescent and young adult as a risk factor for clinical distress, depression, or anxiety in cancer. J Adolesc Young Adult Oncol. 2015, 4:157-73. 10.1089/jayao.2015.0005

6. International Classification of Diseases, 10th Revision (ICD-10), Centers for Disease Control and Prevention. (2001). Accessed: 28/07/2021: https://www.cdc.gov/nchs/data/dvs/icd10fct.pdf.

7. Hamilton BB, Laughlin JA, Fiedler RC, Granger CV: Interrater reliability of the 7-level functional independence measure (FIM). Scand J Rehabil Med. 1994, 26:115-9.

8. Khan MI, Khan JI, Ahmed SI, Ali S. : The epidemiology of stroke in a developing country (Pakistan) . PJNS. 2019, 3:30-44. 10.15406/jnsk.2018.08.00275

9. Putaala J, Metso AJ, Metso TM, et al.: Analysis of 1008 consecutive patients aged 15 to 49 with first-ever ischemic stroke: the Helsinki young stroke registry. Stroke. 2009, 40:1195-203. 10.1161/STROKEAHA.108.529883

10. Pérez-López FR, Larrad-Mur L, Kallen A, Chedraui P, Taylor HS: Gender differences in cardiovascular disease: hormonal and biochemical influences. Reprod Sci. 2010, 17:511-31. 10.1177/1933719110367829

11. O'Donnell MJ, Chin SL, Rangarajan S, et al.: Global and regional effects of potentially modifiable risk factors associated with acute stroke in 32 countries (INTERSTROKE): a case-control study. Lancet. 2016, 10046:761775. 10.1016/S0140-6736(16)30506-2

12. Friedrich V, Bederson JB, Sehba FA: Gender influences the initial impact of subarachnoid hemorrhage: an experimental investigation. PLoS One. 2013, 8:e80101. 10.1371/journal.pone.0080101

13. Derdeyn CP, Zipfel GJ, Albuquerque FC, Cooke DL, Feldmann E, Sheehan JP, Torner JC: Management of brain arteriovenous malformations: a scientific statement for healthcare professionals from the American Heart Association/American Stroke Association. Stroke. 2017, 48:e200-24. 10.1161/STR.0000000000000134

14. Giang KW, Mandalenakis Z, Dellborg M, Lappas G, Eriksson P, Hansson PO, Rosengren A: Long-term risk of hemorrhagic stroke in young patients with congenital heart disease. Stroke. 2018, 49:1155-62. 10.1161/STROKEAHA.117.020032

15. Bennett DA, Krishnamurthi RV, Barker-Collo S, et al.: The global burden of ischemic stroke: findings of the GBD 2010 study. Glob Heart. 2014, 9:107-12. 10.1016/j.gheart.2014.01.001

16. Habibi-Koolaee M, Shahmoradi L, Niakan Kalhori SR, Ghannadan H, Younesi E: Prevalence of stroke risk factors and their distribution based on stroke subtypes in Gorgan: a retrospective hospital-based study2015-2016. Neurol Res Int. 2018, 2018:2709654. 10.1155/2018/2709654

17. Law MR, Morris JK, Wald NJ: Use of blood pressure lowering drugs in the prevention of cardiovascular disease: meta-analysis of 147 randomised trials in the context of expectations from prospective epidemiological studies. BMJ. 2009, 338:b1665. 10.1136/bmj.b1665

18. Ansell BJ: Cholesterol, stroke risk, and stroke prevention. Curr Atheroscler Rep. 2000, 2:92-6. 10.1007/s11883-000-0101-5

19. Rizzo MR, Marfella R, Barbieri M, et al.: Relationships between daily acute glucose fluctuations and cognitive performance among aged type 2 diabetic patients. Diabetes Care. 2010, 33:2169-74. 10.2337/dc100389

20. Ihle-Hansen H, Thommessen B, Wyller TB, Engedal K, Fure B: Risk factors for and incidence of subtypes of ischemic stroke. Funct Neurol. 2012, 27:35-40.

21. Al Dawish MA, Robert AA, Braham R, Al Hayek AA, Al Saeed A, Ahmed RA, Al Sabaan FS: Diabetes Mellitus in Saudi Arabia: A Review of the Recent Literature. Curr Diabetes Rev. 2016, 12:359-68. 10.2174/1573399811666150724095130

22. Arboix A: Cardiovascular risk factors for acute stroke: Risk profiles in the different subtypes of ischemic stroke. World J Clin Cases. 2015, 3:418-29. 10.12998/wjcc.v3.i5.418

23. Chen R, Ovbiagele B, Feng W: Diabetes and stroke: epidemiology, pathophysiology, pharmaceuticals and outcomes. Am J Med Sci. 2016, 351:380-6. 10.1016/j.amjms.2016.01.011

24. Cuadrado-Godia E, Ois A, Roquer J: Heart failure in acute ischemic stroke. Curr Cardiol Rev. 2010, 6:202-13. 10.2174/157340310791658776

25. Han J, Mao W, Ni J, et al.: Rate and determinants of recurrence at 1 year and 5 years after stroke in a lowincome population in rural China. Front Neurol. 2020, 11:2. 10.3389/fneur.2020.00002

26. Suk SH, Sacco RL, Boden-Albala B, Cheun JF, Pittman JG, Elkind MS, Paik MC: Abdominal obesity and risk of ischemic stroke: the Northern Manhattan Stroke Study. Stroke. 2003, 34:1586-92. 10.1161/01.STR.0000075294.98582.2F

27. Coleman ER, Moudgal R, Lang K, Hyacinth HI, Awosika OO, Kissela BM, Feng W: Early Rehabilitation After Stroke: a Narrative Review. Curr Atheroscler Rep. 2017, 19:59. 